\title{
SEOM Clinical Guideline for treatment of muscle-invasive and metastatic urothelial bladder cancer (2016)
}

\author{
M. Lázaro ${ }^{1}$ - E. Gallardo ${ }^{2}$ - M. Doménech ${ }^{3}$ Á. Pinto ${ }^{4}$ A. González del Alba ${ }^{5}$. \\ J. Puente ${ }^{6} \cdot$ O. Fernández ${ }^{7} \cdot$ A. Font ${ }^{8} \cdot$ N. Lainez ${ }^{9} \cdot$ S. Vázquez $^{10}$
}

Received: 13 November 2016/Accepted: 15 November 2016/Published online: 29 November 2016

(C) The Author(s) 2016. This article is published with open access at Springerlink.com

\begin{abstract}
The goal of this article is to provide recommendations for the diagnosis and treatment of muscle-invasive and metastatic bladder cancer. The diagnosis of muscle-invasive bladder cancer is made by pathologic evaluation after transurethral resection. Recently, a molecular classification has been proposed. Staging of muscle-invasive bladder cancer must be done by computed tomography scans of the chest, abdomen and pelvis and classified on the basis of UICC system. Radical cystectomy and lymph node dissection are the treatment of choice. In
\end{abstract}

\author{
M. Lázaro \\ martin.lazaro.quintela@sergas.es \\ E. Gallardo \\ egallardo@tauli.cat \\ M. Doménech \\ mdomenech@althaia.cat \\ Á. Pinto \\ alvaropintomarin@gmail.es \\ A. González del Alba \\ aranzazu.gonzalezdelalba@ssib.es \\ J. Puente \\ docjavip@yahoo.es \\ O. Fernández \\ ovidio.fernandez.calvo@sergas.es \\ A. Font \\ afont@iconcologia.net \\ N. Lainez \\ nuria.lainez.milagro@cfnavarra.es \\ S. Vázquez \\ sergio.vazquez.estevez@sergas.es
}

1 Medical Oncology Department, Hospital Álvaro CunqueiroComplexo Hospitalario Universitario de Vigo, Estrada Clara Campoamor, 34136312 Vigo, Spain muscle-invasive bladder cancer, neoadjuvant chemotherapy should be recommended in patients with good performance status and no renal function impairment. Although there is insufficient evidence for use of adjuvant chemotherapy, its use must be considered when neoadjuvant therapy had not been administered in high-risk patients. Multimodality bladder-preserving treatment in localized disease is an alternative in selected and compliant patients for whom cystectomy is not considered for clinical or personal reasons. In metastatic disease, the first-line

2 Medical Oncology Department, Parc Taulí Sabadell Hospital Universitari, Sabadell, Spain

3 Medical Oncology Department, Althaia, Xarxa Assisencial i Universitària de Manresa, Manresa, Spain

4 Medical Oncology Department, Hospital Universitario La Paz-Idipaz, Madrid, Spain

5 Medical Oncology Department, Hospital Universitario Son Espases, Palma de Mallorca, Spain

6 Medical Oncology Department, Hospital Universitario San Carlos, Madrid, Spain

7 Complexo Hospitalario Universitario de Ourense, Badalona, Spain

8 Medical Oncology Department, Germans Trias i Pujol University Hospital, Badalona, Spain

9 Medical Oncology Department, Complejo Hospitalario de Navarra, Pamplona, Spain

10 Medical Oncology Department, Hospital Universitario Lucus Augusti, Lugo, Spain 
treatment for patients must be based on cisplatin-containing combination. Vinflunine is the only drug approved for use in second line in Europe. Recently, immunotherapy treatment has demonstrated activity in this setting.

Keywords Bladder cancer - Cystectomy · Chemotherapy · Clinical guidelines

\section{Introduction}

According to GLOBOCAN, about 430,000 new bladder cancer cases and 165,000 bladder cancer deaths occurred worldwide in 2012, making it the ninth most common type of cancer for both gender [1].

Europe has one of the highest incidence rates of bladder cancer in the world. According to cancer registry data, the highest incidence rates in men were reported in Southern Europe, particularly in Spain (age-standardized rate $(\mathrm{ASR})=36.7$ per 100,000$)$ and Italy $(\mathrm{ASR}=33.2$ per 100,000). In Spain, around 12,200 new cases are diagnosed every year, with 47 cases per 100,000 men and almost eight cases per 100,000 women [2].

Overall, bladder cancer mortality has been decreasing all over the world except in countries undergoing rapid economic transition [3]. Mortality rates in European men were by far the highest recorded worldwide (e.g., Spain: $\mathrm{ASR}=8.2$ per 100,000 ).

Smoking is recognized as the most important risk factor for urothelial bladder cancer (BC) (ever-smokers are considered to have a 2.5 times higher risk of developing this tumor than nonsmokers) [3] and is estimated to account for $50 \%$ of tumors (former tobacco smoking RR $2.04,95 \%$ CI 1.85-2.25, $p<0.001$; current tobacco smoking RR 3.47, 95\% CI 3.07-3.91, $p<0.001$ when compared to never smokers) [4].
Following smoking, occupational exposure to carcinogens, namely aromatic amines, polycyclic aromatic, hydrocarbons, and chlorinated hydrocarbons is viewed as the second most important risk factor for urothelial BC. There are several medical conditions that may predispose individuals to bladder tumorigenesis: chronic urinary retention and upper tract dilation increase urothelial exposure to carcinogens and carcinogenesis associated with chronic inflammation or schistosomiasis [2].

\section{Methodology}

The SEOM guidelines have been developed with the consensus of ten genitourinary cancer oncologists from SEOM (Spanish Society of Medical Oncology) and SOGUG (Spanish Oncology Genitourinary Group).

To assign a level of levels of evidence and grades of recommendation we have used Table 1 [5].

Statements without grading were considered justified standard clinical practice by the SEOM/SOGUG faculty and experts.

\section{Molecular biology: molecular classification}

Molecular genetic evidence supports the existence of two distinct pathogenetic pathways for bladder cancer development: low-grade papillary superficial tumors (characterized by activation of the receptor tyrosine kinase-Ras pathway, and activating mutations in the HRAS and fibroblast growth factor receptor 3 (FGFR3) genes) and high-grade invasive $\mathrm{BC}$ (characterized by alterations in the p53 and retinoblastoma (RB1) pathways). These genes interact with the Ras-mitogen-activated protein kinase (MAPK) signal transduction pathways [6].

Table 1 Levels of evidence/grades of recommendation

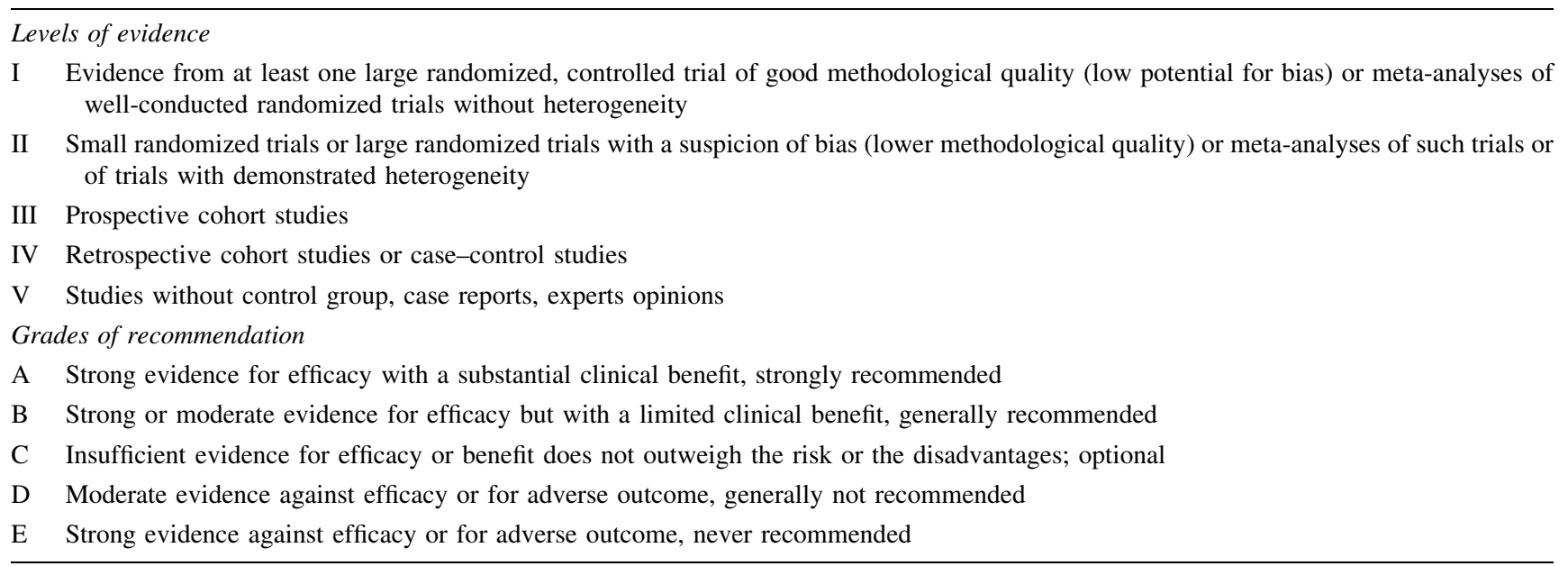


The variability in outcomes of muscle-invasive bladder cancer (MIBC) can be explained by differences in the genetic changes involved in bladder cancer development and progression. The Cancer Genome Atlas Project completed one of the most comprehensive molecular analyses in bladder cancer, examining 131 cases of MIBC [7]. Tumors were histologically categorized and evaluated via whole genome sequencing, whole exome sequencing, DNA copy number, complete mRNA and microRNA expression, DNA methylation, and protein expression and phosphorylation. Many genes were consistently mutated, including TP53, PIK3CA, RB1, FGFR3, and TSC1. In addition, a few pathways were identified as consistently dysregulated. Mutations in the p53/RB tumor suppressor pathway were seen in $93 \%$ of tumors, and alterations in the PI3K/AKT/ mTOR and RTK/RAS signaling were seen in $72 \%$. Finally, alterations that impact epigenetic changes were seen up to $89 \%$ of tumors.

In the last few years, several studies have proposed a molecular classification of bladder cancer based in the whole genome mRNA expression profiling. The molecular subtypes identified in bladder cancer have significant similarities with the molecular classification previously established in breast cancer patients [8]. A group from the University of North Carolina classified high-grade bladder cancer in luminal and basal-like tumors using a 47-gene signature (BASE 47) [9]. TCGA study [7] defined four mRNA expression-based subtypes (cluster I-IV). The cluster I-II correlated with luminal subtype and cluster IIIIV with basal subtype. A third classification proposed by the MD Anderson group identified basal tumors and segregated the luminal subtype in luminal and p53 like tumors [10]. In an expanded cohort of 238 TCGA invasive bladder cancers, four subtypes were identified. Besides luminal and basal subtypes, two new subtypes were proposed related to immune cell infiltration termed "immune undifferentiated" and "luminal immune" characterized by the expression of immune genes (CTLA4 and CD274) [11].

Intrinsic subtypes in MIBC patients are associated with specific clinical-pathological characteristics. Basal MIBC are more prevalent in women and are enriched with squamous pathological features, whereas tumors with a micropapillary histological variant are mainly classified in the luminal subtype [12] Basal tumors were associated with advanced disease with poor prognosis. In contrast, luminal tumors have papillary features that correspond to better outcome when presented at early stage.

Furthermore, molecular classification according to gene expression profile can be correlated with bladder cancer outcome and chemosensitivity. Luminal subtype in BC patients has been associated with chemoresistance. Moreover, only a minority of patients with p53 like tumors responded to neoadjuvant MVAC chemotherapy, suggesting that patients with this molecular subtype should be treated with alternative approaches [10]. In contrast, the greatest benefit of neoadjuvant chemotherapy has been observed in patients with basal subtype [13]. In addition, the evidence of immune cell infiltration in specific bladder cancer subtypes suggests a potential benefit of immune checkpoint agents in these patients. A recently published study showed higher efficacy of atezolizumab in advanced bladder cancer patients with the TCGA luminal cluster II subtype compared with other subtypes [14]. In conclusion, molecular classification of bladder cancer can be a useful tool to select the most adequate therapy for each patient. These advances should be incorporated into the design of clinical trials and progressively into the clinical practice.

\section{Diagnosis and staging}

The symptom that is most frequently presented is hematuria, and irritative urinary symptoms are also common.

Initial diagnosis study is composed of physical examination, complete tests with hemograma and biochemistry, cytoscopy, urinary cytology and an image of the upper urinary tract. The findings of the cytoscopy must be described in detail, including location, size and number of injuries. The fluorescent cytoscopy can detect more tumors, especially in carcinoma in situ and papillary injuries, therefore it is useful in patients on whom a radiotherapy treatment is chosen for.

It is followed by bimanual examination under anesthesia (EUA) and transurethral resection of the bladder (TURBT). This is important to obtain a pathological diagnosis and for MIBC in staging, therefore, it is mandatory to include a representation of the muscular layer. In cases with positive cytology and a normal cystoscopy, an upper urinary tract and prosthetic urethra exam must be carried out.

Histological diagnosis is based on the World Health Organization (WHO) classification [15] and tumor grade is an important factor to determine the potential progression and recurrence of the tumor.

In patients with a MIBC confirmed diagnosis, a computed tomography (CT) of chest, abdomen and pelvis, must be performed as a method of initial staging, including an excretory-phase CT urography for the upper urinary tract study, being MRI also an option. Both are useful for local staging. Routine FDG-PET/CT is not recommended for routine initial staging on MIBC. Bone scan must be performed if there are bone related symptoms or high levels of alkaline phosphatase [16].

Staging must be done according to the norms of the American joint committee on cancer (AJCC) staging manual 7 th edition (Table 2). 
Table 2 TNM staging

TNM staging system [62]

Stage I: T1 N0 M0

Stage II: T2a-T2b N0 M0

Stage III: T3a-T3b, T4a N0 M0

Stage IV: T4b N0 M0

Any T: N1-N3 M0

Any T: Any N M1

\section{Recommendations}

Initial diagnosis study is composed of physical examination cytoscopy, urinary cytology and an image of the upper urinary tract, followed by bimanual EUA and TURBT.

Level of evidence 2. Grade of recommendation B.

$\mathrm{CT}$ of chest, abdomen and pelvis, must be performed as a method of initial staging, including an excretory-phase CT urography for the upper urinary tract study.

Level of evidence: 2. Grade of recommendation B.

\section{Prognostic factors}

The relevance of prognosis classifications is to identify subgroups of patients to decide the most adequate management for every single patient and should be considered for stratification for future trials.

Tumor histologic grade and staging have been recognized as significant prognostic factors for recurrence and progression among patients with nonmetastatic bladder cancer. In addition, pathological response has been proved to be prognostic for patients with muscle-invasive bladder tumors treated with neoadjuvant chemotherapy [17].

At metastatic setting, a prognostic classification was established for first-line chemotherapy after identifying two independent prognostic clinical factors: Karnofsky performance status less than $80 \%$ and the presence of visceral metastases (liver, lung and bone) [18]. Patients can be classified as good, intermediate or bad prognosis after the presence of none, one or both factors (median overall survival: 33, 13.4 and 9.3 months, respectively). This classification was subsequently validated in another independent series from a prospective study [19].

For second-line patients, another prognostic classification was proposed [20]. Three independent prognostic factors in this setting were identified: ECOG performance status more than 0 , hemoglobin value less than $10 \mathrm{~g} / \mathrm{dL}$ and the presence of liver metastases. After internal and external validation, four categories were established from the presence of none, one, two or three factors with median survival times of 14.2, 7.3, 3.8, and 1.7 months. Time from first-line chemotherapy [21] and albumin levels [22] have also proved independent prognostic significance and external validation.

At present, no prognostic biomarkers have been validated for these patients. The indefectible progression after standard chemotherapy and the introduction of new therapeutic approaches deserves further research efforts in this way.

\section{Recommendations}

Use of prognostic classification in first-line chemotherapy. Level of evidence: II. Grade of recommendation: B.

Use of prognostic classification in second-line chemotherapy.

Level of evidence: II. Grade of recommendation: B.

\section{Stage II-III treatment}

\section{Radical cystectomy}

Radical cystectomy (RC) with extended lymphadenectomy, often preceded by neoadjuvant cisplatin-based chemotherapy, and urinary diversion is the gold standard definitive surgical treatment MIBC [23] and involves removal of the bladder, prostate, seminal vesicles, proximal vas deferens and proximal urethra in men, and bladder, uterus, ovaries, fallopian tubes, urethra and part of vagina in women. A randomized [24] trial of roboticassisted laparoscopic versus open radical cystectomy showed no difference in morbidity or length of hospital stay, but longer operative time and increased cost in the robotic group, similar to results from the CORAL study [25] from the UK. Forms of urinary diversion include orthotopic bladder replacement and uretero-ileourethrostomy (Studer and Padovana neobladder) or an incontinent external ostomy with cutaneous ureteroileostomy (Bricker diversion). A glomerular filtration rate of at least $50 \mathrm{~mL} / \mathrm{min}$ is mandatory for continent reservoirs since the kidneys must compensate the metabolic acidosis following incorporation of bowel in the urinary tract [26]. Candidates for a continent urinary diversion should also have normal liver function (risk of hyperammonemia if the reservoir becomes infected), and should not have undergone any previous major bowel resection in the ileocecal area (risk of vitamin B12 deficiency) [26].

Three retrospective cohort studies found regional lymph node dissection to be associated with a lower risk of mortality compared with no lymph node dissection in individuals undergoing radical cystectomy for localized muscle-invasive bladder cancer [27-29]. More extensive 
lymph node dissection was associated with a decreased risk of all-cause or bladder cancer-specific mortality [27]. In one study, dissection in which ten lymph nodes were removed was found to be associated with lower 10 year overall (30.3 vs $39.4 \% ; p<0.001)$ and cancer-specific (38 vs $46 \%$ ) mortality compared with dissection in which $<10$ lymph nodes were removed [27].

\section{Recommendations}

Radical cystectomy with extended lymphadenectomy is the gold standard definitive surgical treatment in muscle-invasive bladder cancer.

Level of evidence: III. Grade of recommendation: A.

\section{Neoadjuvant and adjuvant treatment}

Although surgery may be curative, a large proportion of patients will develop recurrence and will ultimately die of metastatic disease. Several studies have been performed with perioperative chemotherapy, in both neoadjuvant and adjuvant settings.

The major impediment to the use of perioperative chemotherapy in patients with bladder cancer is renal impairment and comorbidities. Around $50 \%$ of patients have a glomerular filtration rate of less than $60 \mathrm{~mL} / \mathrm{min}$, making them ineligible for cisplatin treatment.

\section{Neoadjuvant treatment}

Neoadjuvant chemotherapy has been evaluated in patients with clinical stage T2-T4aN0M0 MIBC who are candidates for $\mathrm{RC}$ or definitive radiotherapy. The rationale for giving chemotherapy before cystectomy or full-dose radiation therapy is to treat micrometastases present at diagnosis, as approximately $50 \%$ of patients diagnosed with MIBC develop metastatic disease within 2 years.

Cisplatin-based combination chemotherapy provides a greater survival benefit than surgery alone in two large, well-designed, randomized trials $[17,30]$ and two metaanalyses $[31,32]$. Despite a 5\% survival benefit at 5 years, the neoadjuvant approach prior to cystectomy has not been widely accepted.

The different neoadjuvant regimens have not been compared in randomized trials, and the ideal cisplatinbased combination chemotherapy has not been established. Methotrexate, vinblastine, doxorubicin, and cisplatin (MVAC) is the best-studied regimen for neoadjuvant chemotherapy. Dose-dense MVAC (DDMVAC) and gemcitabine plus cisplatin (GC) regimens have been also evaluated in several retrospective studies with no substantive difference in the response rate.

\section{Recommendation}

Neoadjuvant chemotherapy is recommended for T2-T4a, cN0 M0 bladder cancer and should always be cisplatinumbased combination therapy.

Level of evidence I. Grade of recommendation A.

Neoadjuvant chemotherapy is not recommended for patients with ECOG PS 2 and/or impaired renal function.

Level of evidence I. Grade of recommendation A.

\section{Adjuvant treatment}

An updated meta-analysis of nine randomized trials, including 945 patients found and overall survival (OS) and disease-free survival (DFS) benefit among those who received cisplatin-based adjuvant chemotherapy [33]. For OS the pooled hazard ratio (HR) across all nine trials was 0.77 (95\% CI $0.59-0.99 ; p=0.049$ ). On the other hand, the pooled HR for DFS was 0.66 (95\% CI $0.45-0.91$; $p=0.014)$. This DFS benefit was more apparent among those with positive nodal involvement $(p=0.010)$.

All the published trials were prematurely terminated and all included enrolled less than 100 patients each. Moreover, two larger randomized clinical trials reported conflicting results (Spanish trial SOGUG 99/01 that enrolled 142 patients [34] and Italian trial that accrued over 180 patients [35]). In the SOGUG trial, HR for intention-to-treat population was 0.37 (95\% CI 0.64-0.22, $p<0.0004$ ) favorable to chemotherapy arm, but the study was prematurely closed. In the Italian trial was no differences in overall survival between adjuvant chemotherapy and control group ( $48 \%$ at 5 years).

In the largest and most recent trial, 284 patients were randomly assigned to either four cycles of adjuvant chemotherapy (immediate treatment) or deferred treatment. The difference in 5-year overall survival was not statistically significant (53.6 vs $47.7 \%$; HR $0.78,95 \%$ CI $0.56-1.08$ ) [36]. In a post hoc exploratory analysis, overall survival was significantly improved in those without lymph node involvement at baseline (79.5 vs $59.0 \%$ ).

\section{Recommendation}

While there is still insufficient evidence for the routine use of adjuvant chemotherapy in clinical practice, it is likely that high-risk patients (extravesical and/or node-positive disease) that have not received neoadjuvant chemotherapy will benefit most from adjuvant chemotherapy.

Level of evidence I. Grade of recommendation A.

Neoadjuvant chemotherapy should still be preferred due to a higher level of evidence and better feasibility.

Level of evidence I. Grade of recommendation A. 


\section{Bladder-sparing treatments}

Bladder-preserving approaches are reasonable alternatives to cystectomy for patients who are unfit for surgery and those who wish to avoid radical surgery.

Clinical criteria helpful in selecting patients for bladder preservation include small tumors size $(\leq 5 \mathrm{~cm})$ without carcinoma in situ, visible complete TURBT, early tumor stage (T2-T3a), no hydronephrosis and no metastatic lymphadenopathy [37].

Options include TURBT alone, TURBT followed by radiotherapy alone, chemotherapy alone or combination of chemotherapy and radiotherapy (trimodality treatment). However, only chemotherapy combined with radiotherapy has been evaluated in prospective randomized comparisons, the other treatment options are still considered to be investigational.

\section{Trimodality treatment}

Trimodality treatment (TMT) include maximal TURBT followed by radiation (40-45 Gy to the pelvis) with concurrent radiosensitizing chemotherapy and additional radiation boost to the bladder (20-25 Gy) if complete response is documented on repeat biopsy. If persistent or recurrent disease is observed at response evaluation $o$ during follow-up, salvage cystectomy is recommended.

Three prospective randomized trials have demonstrated that concurrent chemoradiotherapy is superior to radiotherapy alone. In a randomized trial of 360 patients, radiotherapy with concurrent mitomycin $\mathrm{C}$ and 5-fluorouracil improved 2 year locoregional disease-free survival from $54 \%$ (radiotherapy alone) to $67 \%$, and 5-year overall survival from 35 to $48 \%$ without increasing grade 3-4 acute or late toxicity [38]. In other study, 99 patients were randomized to receive radiation with or without cisplatin and demonstrated an improved local control rate when cisplatin was given [39]. In the third study, 333 patients were randomized to receive radiotherapy alone or radiotherapy plus carbogen and nicotinamide (CON). At 3 years, there was a $13 \%$ improvement in overall survival in favor of combination arm with a $14 \%$ lower risk of death [40]. The optimal combination of radiotherapy and chemotherapy has not been established.

Neoadjuvant chemotherapy in TMT has not been shown to improve survival. A phase III trial compared the efficacy of two cycles of cisplatin, methotrexate and vinblastine followed by concurrent chemoradiotherapy vs concurrent chemoradiotherapy alone. No difference in complete clinical response or 5-year overall survival was observed [41].

Results from several prospective trials have demonstrated the effectiveness of this approach. Five prospective RTOG trials of TMT in 468 patients demonstrates long term outcomes with 5 year and 10-year survival rates of 57 and $37 \%$, respectively, $80 \%$ of patients retained an intact bladder at 5 year with low rates of toxicity [42].

\section{Recommendations}

TURBT alone or radiotherapy alone cannot be recommended as standard treatment.

Level of evidence: II. Grade of recommendation: B.

Trimodality treatment is an alternative in well- informed and compliant patients for whom cystectomy is not considered for clinical or personal reasons.

Level of evidence: I. Grade of recommendation: A.

\section{First-line therapy of locally advanced and metastatic disease}

\section{First-line therapy for "fit" patients}

Combination cisplatin-containing chemotherapy is the standard of care for fit first-line patients with advanced urothelial carcinoma. Both CG (cisplatin and gemcitabine) and MVAC (methotrexate, vinblastine, doxorubicin and cisplatin) are considered as acceptable treatment options in this indication. There are no clear differences in efficacy between these two schedules, but toxicity seems to be higher with MVAC. In a comparative randomized trial [43], median survival and 5-year survival were 14 months and $13 \%$ for GC, compared to 15.2 months and $15.3 \%$ for MVAC. However, CG has a better safety profile, with a lower incidence of febrile neutropenia, oral mucositis and alopecia, and therefore it is usually the preferred choice for first-line therapy.

High-dose intensity MVAC (HD-MVAC), administered every two weeks along with GCSF support, allows the delivery of twice the dose of cisplatin and doxorubicin. Compared with standard-dose MVAC [44], HD-MVAC showed a higher complete response rate ( $21 \mathrm{vs} 9 \% ; p=0.009$ ) and longer PFS (9.1 vs 8.2 months; $p=0.04$ ), with fewer dose delays and a better toxicity profile, but without differences in OS. HDMVAC should only be considered in selected populations and in centers with experience using this schedule, as it has not been compared with standard CG.

Adding a taxane to standard CG has also been explored in this setting. The combination of paclitaxel plus $\mathrm{CG}$ (PCG) did not show differences in PFS or OS in a phase III trial, and toxicity was higher for the triplet [45].

\section{First-line therapy for "unfit" patients}

A significant percentage of patients with advanced urothelial cancer are considered "unfit" for cisplatin-based chemotherapy, based on the following criteria: Eastern 
Cooperative Oncology Group (ECOG) performance status (PS) 2 or Karnofsky index 60-70\%; creatinine clearance $<60 \mathrm{~mL} / \mathrm{min}$; loss of hearing and/or peripheral neuropathy $\geq$ grade 2 according to the Common Terminology Criteria for Adverse Events version 4.0; or New York Heart Associations Class III heart failure [46]. There is no clear standard therapy for these patients, and the most usual procedure is changing cisplatin for carboplatin, therefore avoiding renal toxicity and improving tolerance to the schedule.

A phase II/III trial comparing the activity of carboplatin/ gemcitabine $(\mathrm{CaG})$ with that of carboplatin/methotrexate/ vinblastine (CaMVi) in 178 unfit patients found similar efficacy for the two regimens [47], with a median OS of 9.3 and 8.1 months, respectively, and with a lower toxicity for $\mathrm{CaG}$. Some other studies have tested the role of non-platinum combinations, such as paclitaxel-gemcitabine, which show encouraging activity, with response rates ranging 40-60\%, but also considerable toxicity. Finally, for frail patients, monotherapy can also be an option, mainly with paclitaxel, gemcitabine or vinflunine, but with limited results.

\section{Recommendations}

For first-line fit patients, both CG and MVAC are considered standard options. CG is preferred over MVAC mainly due to a better safety profile.

Level of evidence: 1. Grade of recommendation: A.

For first-line unfit patients, $\mathrm{CaG}$ should be considered the preferred treatment option.

Level of evidence: 1. Grade of recommendation: A.

\section{Second line}

We have limited treatment options for this scenario. Most of the chemotherapy agents have been tested in phase 2 studies. Paclitaxel, docetaxel, oxaliplatin, pemetrexed, nabpaclitaxel, ifosfamida, among others, have a response rate of around $20 \%$, without having proven a global survival benefit [48]. Combined use of chemotherapy agents increases the response rate and DFS [49].

Vinflunine, a third generation vinca alkaloid, showed a benefit in overall survival in eligible population during a phase 3 study, compared against best supportive care (BSC) (although not in the intended treatment population) and it has been approved by the EMA in this indication (IB) [50].

It is recommended, if possible, the inclusion of these patients in the clinical studies.

\section{Recommendations}

For patients who progress after platinum based therapy offer vinflunine. Treatment in a clinical trial as an alternative.
Level of evidence I. Grade of recommendation B.

\section{New drugs and immunotherapy agents}

Advances in the understanding of the molecular mechanisms of UBC have led to many studies to evaluate targeted therapies. Potential actionable genomic alterations, including activating mutations and RNA expression changes involving the PI3K/AKT/mTOR and RTK/RAS pathways have been frequently detected in the TCGA and other studies [7, 11, 51] Several studies have identified significant activity of targeted therapies in patients with these genomic alterations. For example, patients with mutations in PIK3CA (about $17 \%$ of tumors) can be sensitive to PI3K inhibitors, whereas patients with mutation/amplification of ERBB2 (about $9 \%$ of tumors) or ERBB3 mutations (about $6 \%$ of tumors) can respond to ERBB tyrosine kinase inhibitors, as has been recently demonstrated [52]. Moreover, inactivating mutations of genes involved in DNA repair pathways have also been identified in MIBC patients. Somatic mutations of ERCC2, a crucial gene of the nucleotide excision repair pathway, were found in $6-18 \%$ of urothelial tumors. These mutations have been associated with response to neoadjuvant cisplatinbased chemotherapy in MIBC patients [7, 11, 53, 54].

Immune checkpoint inhibition for cancer treatment is an area of growing research and recent studies have demonstrated that upregulation of PD-L1 is an important mechanism of immune escape in NMIBC. Overexpression of PDL1 in UC correlates with high-grade disease and worse clinical outcome. Remarkable efficacy and safety was seen in a phase I expansion cohort of 67 patients with heavily pretreated metastatic bladder cancer. Patients received $15 \mathrm{mg} / \mathrm{kg}$ of MPDL3280A (atezolizumab), a human monoclonal antibody to PD-L1, every 3 weeks. 89 response rates were reported by PD-L1 positivity status, defined as $5 \%$ or higher of tumor-infiltrating immune cells staining for PD-L1 by IHC [55]. In this study, $27 \%$ of tumors were IHC 2- or 3-positive, as defined by expression of PD-L1 on tumorinfiltrating immune cells. The overall response rate for all patients by response evaluation criteria in solid tumors (RECIST) v1.1 was 26\%, and was even more remarkable (43\%) among patients with PD-L1+ tumor-infiltrating cells. Even among patients whose tumor-infiltrating immune cells were PD-L1-, the response rate was $11 \%$ as measured by RECIST v1.1. The median time to first response was 42 days (range 38-85 days). Based on these results, MPDL3280A received breakthrough designation by the FDA in June 2014. Recently, several other immunotherapies agents such nivolumab [56], pembrolizumab [57], avelumab [58] or durvalumab [59] have shown promising data in different phase I/II trials; and a phase II trial with atezolizumab in platinumtreated patients showed an ORR $16 \%$ (28\% IC2/3 PDL1 subgroup) and an overall survival of 7.9 months (11.9 IC2/3 
PDL1 subgroup) [60]. Another multiple PD-1/PDL-1 agents are currently being tested alone or in combination in advanced/refractory UC. Many more trials are in development in NMIBC.

\section{Follow-up}

There is no data about the best follow-up strategy. Because most recurrences will develop within 24 months, the approach should be an oncological surveillance more intensive in this period of time: history, physical examination, urine cytology, liver and renal function tests, and electrolytes every 3 months for the first year, every 6 months for the second and third years, and then annually. CT imaging is reasonably performed every 6 months for the first 3 years (every 3 months if $\mathrm{N}+$ the first year), then annually to year 5 . After year 5 they should be performed only as clinically indicated $[37,61]$.

\section{Recommendation}

For patients with MIBC a follow-up must be offered. Level of evidence V. Grade of recommendation B.

\section{Compliance with ethical standards}

Conflict of interest The authors have declared that they have no potential conflicts of interest related to the publication of this manuscript.

Open Access This article is distributed under the terms of the Creative Commons Attribution 4.0 International License (http://crea tivecommons.org/licenses/by/4.0/), which permits unrestricted use, distribution, and reproduction in any medium, provided you give appropriate credit to the original author(s) and the source, provide a link to the Creative Commons license, and indicate if changes were made.

\section{References}

1. Ferlay J, Soerjomataram I, Dikshit R, Eser S, Mathers C, Rebelo M, et al. Cancer incidence and mortality worldwide: sources, methods and major patterns in GLOBOCAN 2012. Int J Cancer. 2015;136:E359-86.

2. Burger M, Catto JWF, Dalbagni G, Grossman HB, Herr H, Karakiewicz P, et al. Epidemiology and risk factors of urothelial bladder cancer. Eur Urol. 2013;2:234-41.

3. Babjuk M. Trends in bladder cancer incidence and mortality: success or disappointment? Eur Urol. 2016. doi:10.1016/j.eururo.2016.06.040.

4. Cumberbatch MG, Rota M, Catto JWF, La Vecchia C. The role of tobacco smoke in bladder and kidney carcinogenesis: a comparison of exposures and meta-analysis of incidence and mortality risks. Eur Urol. 2016;70:458-66.

5. Dykewicz CA, Centers for Disease Control and Prevention (U.S.), Infectious Diseases Society of America, American Society of Blood and Marrow Transplantation. Summary of the guidelines for preventing opportunistic infections among hematopoietic stem cell transplant recipients. Clin Infect Dis. 2001;33:139-44.

6. Netto GJ. Molecular biomarkers in urothelial carcinoma of the bladder: are we there yet? Nat Rev Urol. 2012;9:41-51.

7. Cancer Genome Atlas Research Network. Comprehensive molecular characterization of urothelial bladder carcinoma. Nature. 2014;507:315-22.
8. Perou CM, Sørlie T, Eisen MB, van de Rijn M, Jeffrey SS, Rees CA, et al. Molecular portraits of human breast tumours. Nature. 2000;406:747-52.

9. Damrauer JS, Hoadley KA, Chism DD, Fan C, Tiganelli CJ, Wobker SE, et al Intrinsic subtypes of high-grade bladder cancer reflect the hallmarks of breast cancer biology. Proc Natl Acad Sci USA. 2014;111:3110-5.

10. Choi W, Porten S, Kim S, Willis D, Plimack ER, Hoffman-Censits J, et al Identification of distinct basal and luminal subtypes of muscle-invasive bladder cancer with different sensitivities to frontline chemotherapy. Cancer Cell 2014;25:152-65.

11. Kim J, Akbani R, Creighton CJ, Lerner SP, Weinstein JN, Getz G, et al Invasive bladder cancer: genomic insights and therapeutic promise. Clin Cancer Res. 2015;21:4514-24.

12. Guo CC, Dadhania V, Zhang L, Majewski T, Bondaruk J, Sykulski M, et al Gene expression profile of the clinically aggressive micropapillary variant of bladder cancer. Eur Urol. 2016;70:611-20.

13. McConkey DJ, Choi W, Shen Y, Lee IL, Porten S, Matin SF, et al. A prognostic gene expression signature in the molecular classification of chemotherapy-naïve urothelial cancer is predictive of clinical outcomes from neoadjuvant chemotherapy: a phase 2 trial of dose-dense methotrexate, vinblastine, doxorubicin, and cisplatin with bevacizumab in urothelial cancer. Eur Urol. 2016;69:855-62.

14. Rosenberg JE, Hoffman-Censits J, Powles T, van der Heijden MS, Balar AV Necchi A, et al. Atezolizumab in patients with locally advanced and metastatic urothelial carcinoma who have progressed following treatment with platinumbased chemotherapy: a single-arm, multicentre, phase 2 trial. Lancet. 2016;387:1909-20.

15. Humphrey PA, Moch H, Cubilla AL, Ulbright TM, Reuter VE. The 2016 WHO classification of tumours of the urinary system and male genital organs-part B: prostate and bladder tumours. Eur Urol. 2016;70:106-19.

16. Witjes JA, Compérat E, Cowan NC, De Santis M, Gakis G, Lebret T, et al. EAU guidelines on muscle-invasive and metastatic bladder cancer: summary of the 2013 guidelines. Eur Urol. 2014;65:778-92.

17. Grossman HB, Natale RB, Tangen CM, Speights VO, Vogelzang NJ, Trump DL, et al. Neoadjuvant chemotherapy plus cystectomy compared with cystectomy alone for locally advanced bladder cancer. $N$ Engl J Med. 2003;349:859-66.

18. Bajorin DF, Dodd PM, Mazumdar M, Fazzari M, McCaffrey JA, Scher HI, et al. Long-term survival in metastatic transitional-cell carcinoma and prognostic factors predicting outcome of therapy. J Clin Oncol. 1999;17:3173-81.

19. Bellmunt J, Albanell J, Paz-Ares L, Climent MA, González-Larriba JL, Carles J et al. Pretreatment prognostic factors for survival in patients with advanced urothelial tumors treated in a phase I/II trial with paclitaxel, cisplatin, and gemcitabine. Cancer. 2002;95:751-7.

20. Bellmunt J, Choueiri TK, Fougeray R, Schutz FAB, Salhi Y, Winquist E, et al. Prognostic factors in patients with advanced transitional cell carcinoma of the urothelial tract experiencing treatment failure with platinum-containing regimens. J Clin Oncol. 2010;28:1850-5.

21. Sonpavde G, Pond GR, Fougeray R, Choueiri TK, Qu AQ, Vaughn DJ, et al Time from prior chemotherapy enhances prognostic risk grouping in the secondline setting of advanced urothelial carcinoma: a retrospective analysis of pooled, prospective phase 2 trials. Eur Urol. 2013:63:717-23.

22. Sonpavde G, Pond GR, Rosenberg JE, Bajorin DF, Choueiri TK, Necchi A, et al. Improved 5-factor prognostic classification of patients receiving salvage systemic therapy for Advanced urothelial carcinoma. J Urol. 2016;195:277-82.

23. Gakis G, Efstathiou J, Lerner SP, Cookson MS, Keegan KA, Guru KA, et al. ICUD-EAU International Consultation on Bladder Cancer 2012: radical cystectomy and bladder preservation for muscle-invasive urothelial carcinoma of the bladder. Eur Urol. 2013;63:45-57.

24. Bochner BH, Dalbagni G, Sjoberg DD, Silberstein J, Keren Paz GE, Donat SM, et al. Comparing open radical cystectomy and robot-assisted laparoscopic radical cystectomy: a randomized clinical trial. Eur Urol. 2015;67:1042-50.

25. Khan MS, Gan C, Ahmed K, Ismail AF, Watkins J, Summers JA, et al. A singlecentre early phase randomised controlled three-arm trial of open, robotic, and laparoscopic radical cystectomy (CORAL). Eur Urol. 2016;69:613-21.

26. Roth B, Thalmann GN. Standard cystectomy fits all: truth or myth? Transl Androl Urol. 2015;4:254-60.

27. Abdollah F, Sun M, Schmitges J, Djahangirian O, Tian Z, Jeldres C, et al. Stagespecific impact of pelvic lymph node dissection on survival in patients with nonmetastatic bladder cancer treated with radical cystectomy. BJU Int. 2012;109:1147-54.

28. Herr HW, Bochner BH, Dalbagni G, Donat SM, Reuter VE, Bajorin DF. Impact of the number of lymph nodes retrieved on outcome in patients with muscle invasive bladder cancer. J Urol. 2002;167:1295-8.

29. Konety BR, Joslyn SA, O'Donnell MA. Extent of pelvic lymphadenectomy and its impact on outcome in patients diagnosed with bladder cancer: analysis of data from the surveillance, epidemiology and end results program data base. J Urol. 2003;169:946-50.

30. International Collaboration of Trialists, Medical Research Council Advanced Bladder Cancer Working Party (now the National Cancer Research Institute Bladder Cancer Clinical Studies Group), European Organisation for Research and Treatment of Cancer Genito-Urinary Tract Cancer Group, Australian Bladder Cancer Study Group, National Cancer Institute of Canada Clinical 
Trials Group, Finnbladder, et al. International phase III trial assessing neoadjuvant cisplatin, methotrexate, and vinblastine chemotherapy for muscle-invasive bladder cancer: long-term results of the BA06 30894 trial. J Clin Oncol. 2011;29:2171-7.

31. Advanced Bladder Cancer (ABC). Meta-analysis Collaboration. Neoadjuvant chemotherapy in invasive bladder cancer: update of a systematic review and meta-analysis of individual patient data advanced bladder cancer (ABC) metaanalysis collaboration. Eur Urol. 2005;48:202-5.

32. Collaboration ABCAM-A. Neoadjuvant chemotherapy in invasive bladder cancer: a systematic review and meta-analysis. Lancet. 2003:361:1927-34

33. Leow JJ, Martin-Doyle W, Rajagopal PS, Patel CG, Anderson EM, Rothman AT, et al. Adjuvant chemotherapy for invasive bladder cancer: a 2013 updated systematic review and meta-analysis of randomized trials. Eur Urol. 2014;66:42-54

34. Paz-Ares LG, Solsona E, Esteban E, Saez A, Gonzalez-Larriba J, Anton A, et al. Randomized phase III trial comparing adjuvant paclitaxel/gemcitabine/cisplatin (PGC) to observation in patients with resected invasive bladder cancer: results of the Spanish Oncology Genitourinary Group (SOGUG) 99/01 study. J Clin Oncol (ASCO Meeting Abstracts). 2010;28(18_suppl):LBA4518.

35. Cognetti F, Ruggeri EM, Felici A, Gallucci M, Muto G, Pollera CF, et al Adjuvant chemotherapy with cisplatin and gemcitabine versus chemotherapy at relapse in patients with muscle-invasive bladder cancer submitted to radical cystectomy: an Italian, multicenter, randomized phase III trial. Ann Oncol. 2012;23:695-700.

36. Sternberg CN, Skoneczna I, Kerst JM, Albers P, Fossa SD, Agerbaek M, et al. Immediate versus deferred chemotherapy after radical cystectomy in patients with pT3-pT4 or N+ M0 urothelial carcinoma of the bladder (EORTC 30994): an intergroup, open-label, randomised phase 3 trial. Lancet Oncol. 2015;16:76-86.

37. Morales R, Font A, Carles J, Isla D. SEOM clinical guidelines for the treatment of invasive bladder cancer. Clin Transl Oncol. 2011;13:552-9.

38. James ND, Hussain SA, Hall E, Jenkins P, Tremlett J, Rawlings C, et al. Radiotherapy with or without chemotherapy in muscle-invasive bladder cancer. N Engl J Med. 2012;366:1477-88.

39. Coppin CM, Gospodarowicz MK, James K, Tannock IF, Zee B, Carson J, et al. Improved local control of invasive bladder cancer by concurrent cisplatin and preoperative or definitive radiation. The National Cancer Institute of Canada Clinical Trials Group. J Clin Oncol. 1996;14:2901-7.

40. Hoskin PJ, Rojas AM, Bentzen SM, Saunders MI. Radiotherapy with concurrent carbogen and nicotinamide in bladder carcinoma. J Clin Oncol. 2010;28:4912-8.

41. Shipley WU, Winter KA, Kaufman DS, Lee WR, Heney NM, Tester WR, et al. Phase III trial of neoadjuvant chemotherapy in patients with invasive bladder cancer treated with selective bladder preservation by combined radiation therapy and chemotherapy: initial results of Radiation Therapy Oncology Group 89-03. J Clin Oncol. 1998;16:3576-83.

42. Mak RH, Hunt D, Shipley WU, Efstathiou JA, Tester WJ, Hagan MP, et al Long-term outcomes in patients with muscle-invasive bladder cancer after selective bladder-preserving combined-modality therapy: a pooled analysis of Radiation Therapy Oncology Group protocols 8802, 8903, 9506, 9706, 9906, and 0233. J Clin Oncol. 2014;32:3801-9.

43. von der Maase H, Sengelov L, Roberts JT, Ricci S, Dogliotti L, Oliver T, et al. Long-term survival results of a randomized trial comparing gemcitabine plus cisplatin, with methotrexate, vinblastine, doxorubicin, plus cisplatin in patients with bladder cancer. J Clin Oncol. 2005;23:4602-8.

44. Sternberg CN, de Mulder P, Schornagel JH, Theodore C, Fossa SD, van Oosterom AT, et al. Seven year update of an EORTC phase III trial of high-dose intensity M-VAC chemotherapy and G-CSF versus classic M-VAC in advanced urothelial tract tumours. Eur J Cancer. 2006;42:50-4.

45. Bellmunt J, von der Maase H, Mead GM, Skoneczna I, De Santis M, Daugaard $\mathrm{G}$, et al. Randomized phase III study comparing paclitaxel/cisplatin/gemcitabine and gemcitabine/cisplatin in patients with locally advanced or metastatic urothelial cancer without prior systemic therapy: EORTC Intergroup Study 30987. J Clin Oncol. 2012;30:1107-13.

46. Galsky MD, Hahn NM, Rosenberg J, Sonpavde G, Hutson T, Oh WK, et al. Treatment of patients with metastatic urothelial cancer "unfit" for cisplatinbased chemotherapy. J Clin Oncol. 2011;29:2432-8.

47. De Santis M, Bellmunt J, Mead G, Kerst JM, Leahy M, Maroto P, et al. Randomized phase II/III trial assessing gemcitabine/carboplatin and methotrexate/carboplatin/vinblastine in patients with advanced urothelial cancer who are unfit for cisplatin-based chemotherapy: EORTC study 30986. J Clin Oncol. 2012;30:191-9.

48. Oing C, Rink M, Oechsle K, Seidel C, von Amsberg G, Bokemeyer C. Secondline chemotherapy for advanced and metastatic urothelial carcinoma-vinflunine and beyond: a comprehensive review of the current literature. J Urol. 2016;195:254-63.

49. Raggi D, Miceli R, Sonpavde G, Giannatempo P, Mariani L, Galsky MD, et al Second-line single-agent versus doublet chemotherapy as salvage therapy for metastatic urothelial cancer: a systematic review and meta-analysis. Ann Oncol. 2016;27:49-61.

50. Bellmunt J, Théodore C, Demkov T, Komyakov B, Sengelov L, Daugaard G, et al. Phase III trial of vinflunine plus best supportive care compared with best supportive care alone after a platinum-containing regimen in patients with advanced transitional cell carcinoma of the urothelial tract. J Clin Oncol. 2009;27:4454-61.

51. Iyer G, Al-Ahmadie H, Schultz N, Hanrahan AJ, Ostrovnaya I, Balar AV, et al Prevalence and co-occurrence of actionable genomic alterations in high-grade bladder cancer. J Clin Oncol. 2013;31:3133-40.

52. Choudhury NJ, Campanile A, Antic T, Yap KL, Fitzpatrick CA, Wade JL, et al Afatinib activity in platinum-refractory metastatic urothelial carcinoma in patients with ERBB alterations. J Clin Oncol. 2016;34:2165-71.

53. Van Allen EM, Mouw KW, Kim P, Iyer G, Wagle N, Al-Ahmadie H, et al. Somatic ERCC2 mutations correlate with cisplatin sensitivity in muscle-invasive urothelial carcinoma. Cancer Discov. 2014;4:1140-53.

54. Aragon-Ching JB. Is there an optimal treatment sequencing strategy for metastatic castration-resistant prostate cancer? Future Oncol. 2013;9:619-22.

55. Powles T, Eder JP, Fine GD, Braiteh FS, Loriot Y, Cruz C, et al. MPDL3280A (anti-PD-L1) treatment leads to clinical activity in metastatic bladder cancer. Nature. 2014;515:558-62.

56. Sharma P, Bono P, Kim JV, Spiliopoulou P, Calvo E, Pillai RN, et al. Efficacy and safety of nivolumab monotherapy in metastatic urothelial cancer (mUC): results from the phase I/II CheckMate 032 study. J Clin Oncol (ASCO Meeting Abstracts). 2016;34(Suppl):4501.

57. Plimack ER, Bellmunt J, Gupta S. Pembrolizumab (MK-3475) for advanced urothelial cancer: updated results and biomarker analysis from KEYNOTE-012. J Clin Oncol (ASCO Meeting Abstracts). 2015;33(Suppl):4572.

58. Apolo AB, Infante JR, Hamid O, Patel MR, Wang D, Kelly K, et al. Avelumab (MSB0010718C; anti-PD-L1) in patients with metastatic urothelial carcinoma from the JAVELIN solid tumor phase $1 \mathrm{~b}$ trial: analysis of safety, clinical activity, and PD-L1 expression. J Clin Oncol (ASCO Meeting Abstracts). 2016;34(suppl):4514

59. Massard C, Gordon MS, Sharma S, Rafii S, Wainberg ZA, Luke J, et al. Safety and efficacy of durvalumab (MEDI4736), an anti-programmed cell death ligand1 immune checkpoint inhibitor, in patients with advanced urothelial bladder cancer. J Clin Oncol. 2016;34:3119-25.

60. Dreicer R, Hoffman-Censits JH, Flaig TW, Grande E, Balmanoukian AS, von Amsberg $G$, et al. Updated efficacy and $>1-y$ follow up from IMvigor210: atezolizumab (atezo) in platinum (plat) treated locally advanced/metastatic urothelial carcinoma (mUC). J Clin Oncol (ASCO Meeting Abstracts). 2016;34(suppl):4515.

61. Cagiannos I, Morash C. Surveillance strategies after definitive therapy of invasive bladder cancer. Can Urol Assoc J. 2009;3(6 Suppl 4):S237-42.

62. Edge SB, Byrd DR, Compton CC, Fritz AG, Greene FL. American joint committee on cancer (AJCC) staging manual. 7th ed. Berlin: Springer; 2010. 